\title{
The Role of the Unconscious in Marnie and Three Colours: Blue
}

\author{
By Veronica Johnson
}

Fall 2008 Issue of KINEMA

\section{THE ROLE OF THE UNCONSCIOUS IN MARNIE AND THREE COLOURS: BLUE}

The unconscious completely eludes that circle of certainties by which man recognises himself as ego. There is something outside this field which has every right to speak as I, and which makes this right manifest by coming into the world speaking as an I. It is precisely what is most misconstrued by the domain of the ego which, in analysis, comes to be formulated as properly speaking being the I.

Jacques Lacan $^{(1)}$

Film excels at documenting the life of the ego. It is a medium which depends on the portrayal of the conscious actions of the protagonists for its appeal. The narrative of a film is unfolded through these actions. Anything which is "outside this field" of conscious action is more difficult to capture on film; thought processes, imaginings, dreams and wishes present filmmakers with particular problems. The traditional solution to these problems has been the use of the voiceover to capture that which is felt or experienced by the protagonist but which does not manifest itself in visible action. The voice-over however, is generally considered to be uncinematic; it is considered to dictate to the audience, to tell the audience what is happening. Whereas the dominant method of film is to show the audience what is happening. Thus, filmmakers who wish to show what is "outside this field" of consciousness and who wish to do so without the use of voice-over have been forced to invent new methods of filmmaking. Methods which adhere to the criteria of showing a story as is traditional in the medium, while at the same time manage to capture the way in which anything which is outside consciousness presents itself to consciousness as elusive and mysterious.

This article will examine two films; Alfred Hitchcock's Marnie from 1964 and Krzysztof Kielowski's Three Colours: Blue from 1993 in order to discuss the way in which the unconscious - that which is "outside this field" of consciousness - is shown in these films and the effect that this showing has on the narrative of these films. It will contend that the methods used to show the unconscious in these two films appear at first to be ways of subverting the narrative of these films, as each appearance of the unconscious stops the narrative and this disruption of the narrative causes the viewer to initially distrust the narrative. However, the appearance of the unconscious, which initially seems to subvert the narrative, is revealed at the end of these films to have been the main source of narrative agency. These are films of and about the unconscious.

In Marnie and Three Colours: Blue the aspect of the unconscious that is presented is that of repression. The type of repression represented in these films is that which occurs after a traumatic event. Both Marnie, in Hitchcock's film of the same name, and Julie in Three Colours: Blue have experienced a traumatic event. Marnie, as a terrified child helps to kill a sailor that she believes is attacking her mother. Julie loses her husband and daughter in a car crash of which she is the only survivor. Julie and Marnie then repress these traumatic events. Marnie erases the event from her memory entirely; as the film begins she is a young woman with no conscious knowledge of this significant event from her past. Julie actively represses the loss of her family, refusing to mourn or grieve their deaths, in parallel to this repression she also represses her creative ability, before the car crash she composed music with her husband. According to classical psychoanalysis, most acts of repression are actually unsuccessful in their attempts to keep the memory of the painful event repressed completely. What usually happens is that the repressed returns, often in a manner that is extremely disruptive to normal life. This is the case with both Marnie and Julie. They both endure puzzling and painful occurrences of the "return of the repressed." It is these instances of the "return of the repressed" that appear initially to subvert the narrative of these films. Once the repressed has returned the only way that it's disruptive effect can be eliminated is for the original trauma to be revisited.

The narrative of repression then is as follows: the occurrence of a traumatic event, the repression of the knowledge of that event, the "return of the repressed" and a revisiting of the original event in order to come to terms with the event itself and its repercussions. This account of the progression of repression is an 
account that looks at repression from the point of view of the unconscious. From the point of view of the ego or consciousness, a person only becomes aware of repression through the effects of the "return of the repressed." It has a disturbing effect, as the person in question has successfully blocked all knowledge of the original traumatic event from their consciousness, they have no means of understanding either the origin or the significance of the events which announce the "return of the repressed."

Film tells the story from the point of view of consciousness. Both Marnie and Three Colours: Blue follow this tradition. What is presented to the audience in these films is the story of the conscious acts of the protagonists and those of the other characters in the films. We, as viewers follow the narrative as it is carried out through these acts. However, as both these films are about the unconscious, we are like the protagonists, shocked by the appearance of the unconscious through the events marked by the "return of the repressed." These events stop the traditional cinematic narrative; the narrative of consciousness puzzle and bewilder both the protagonist and the viewer. From the point of view of the spectator, the occurrence of these events seems to signal a loss of narrative cohesion.

The extent to which the narrative is disrupted is slightly different for each film. This difference comes from the fact that the viewer is shown Julie's trauma at the beginning of Three Colours: Blue, whereas Marnie's trauma is only revealed at the end of Hitchcock's film. In Three Colours: Blue our knowledge of Julie's trauma leads us to suspect that the "return of the repressed" that occurs in this film is connected to this trauma and to the character's refusal to openly mourn her loss. However, the first appearance of the "return of the repressed" still comes as a shock to the viewer as despite our knowledge of her trauma, the moment of the return of the repressed is given no context within the film. As viewers, we may suspect that these moments have something to do with her trauma, but Kielowski never gives us enough information to connect them directly to each other. Also, as mentioned previously, Julie represses her musical creativity at the same time and the audience does not become aware of this fact until the final act of the film.

With Marnie the disruption to the narrative of the film is much greater, the impact on the audience of these disruptions more upsetting. As we are unaware of Marnie's trauma we are left completely without context for these intrusions into the narrative flow of the film. The fact of knowing or not knowing the trauma undergone by both characters affects the degree of disruption of the narrative that occurs in these films.

Despite this difference of degree, in both films the first time the "return of the repressed" intrudes upon the narrative is the most shocking. Up to this point both films have been fairly conventional in their exposition of the story. In fact, the first appearance of the unconscious occurs just as the audience has begun to settle into the film; the main characters have been introduced, the story has been set up, the "world" of the film has been established. It is at this point that both Kielowski and Hitchcock disturb the audience's sense of security with the narrative by stopping it suddenly with the appearance of the unconscious. This sudden appearance shakes up the audience, it creates a sense of distrust in the narrative, as the visual (and in the case of Three Colours: Blue, aural) power of this intrusion signifies to the audience that an important event has just taken place. However, immediately after this event, the narrative continues as before, making no reference to this event. It thus initially appears to the audience that this event has no narrative significance.

Despite the fact that the unconscious initially seems to have no impact on the narrative of these films, it continues to appear on screen. Time after time the narrative is stopped by the appearance of the unconscious. While initially the audience only registered the fact that these moments were significant, their continuous repetition, combined with the extra knowledge we have gained of the protagonist as the film develops, leads the audience to begin to understand their significance. By the second act of both these films we are aware that Marnie and Julie are troubled women. Julie has isolated herself from friends and family and constantly rejects everyone who tries to engage with her. Marnie has accepted an offer of marriage from her boss who has caught her stealing from his company. While she agrees that this marriage will protect her from prosecution and possibly even prostitution as is hinted in the marriage proposal, it is clear that a part of Marnie would prefer both of these alternatives to her husband's insistent attempts to get her to discover what lies at the root of her troubles. Now that we are aware that these characters are suffering, that they are straining to support the facade of an outer life that is crumbling before their very eyes, it becomes easier for us to see the appearance of the unconscious as a part of the narrative rather than a rupture in the narrative at this point. 
The significance of these appearances of the unconscious is only made clear in the final act of both films. Hitchcock shows us the original traumatic event from the point of view Marnie as a child. Kielowski shows us Julie embracing her creativity and finally beginning to mourn. Thus what appeared to have been a rupture in the narrative is revealed to have been a cinematic method of representing the unconscious in a way that imitates how the unconscious itself ruptures consciousness at those moments when the repressed returns. Robin Wood describes the significance of these moments in Marnie as follows:

Because of her traumatic experience, the colour red, seen either under conditions of great tension or in a way that directly evokes the experience itself..., acts as a release mechanism for Marnie's suppressed tensions - the tensions that are with her, to some extent, continually. Its immediate effect is to provoke a sort of hysterical swoon, a panic reaction, as the terrifying, buried memory forces itself dangerously near the surface of consciousness, which in turn produces a sense of unreality. Now, Hitchcock...doesn't want merely to show us a woman caught in this condition, he wants to convey to us the feeling of the condition itself - wants us to experience it directly as Marnie experiences it, as far as that is possible. What better, simpler, more beautifully economical and direct way than by these red flashes that suffuse the whole screen, filling us, too, with a feeling of panic (we know no more than Marnie what they mean), conveying this sense of being plunged abruptly, arbitrarily, into unreality? ${ }^{(2)}$

It is only at the end of Marnie, and at the end of Three Colours: Blue, that we realise what initially appeared as disruptions in the narrative were in fact moments of vital importance to the development of the narrative. As Wood states, these moments are when "the terrifying, buried memory forces itself dangerously near the surface of consciousness, which in turn produces a sense of unreality."They are moments of "unreality" for the protagonist, that is, moments of a reality that consciousness is unaware of.

At the close of both films, when we watch the protagonists confront their respective traumas, Julie through embracing her musical creativity and opening herself to love again, Marnie through remembering the moment from childhood when she helped kill the sailor, we fully realise that those moments of apparent disruption in the body of the film were in fact messages from the unconscious.

\section{Notes}

1. Lacan, Jacques. The Seminar of Jacques Lacan. Book 11: The Ego in Freud's Theory and in the Technique of Psychoanalysis 1954-1955. Ed. Jacques-Alain Miller. Trans. Sylvana Tomaselli. W. W. Norton and Co. New York, 1988.

2. Wood, Robin. Hitchcock's Films Revisited. Columbia University Press, 2002.

\section{Author Information}

Veronica JOHNSON is a PhD student at the Huston School of Film \& Digital Media at the National University of Ireland, Galway. Her research involves the representation and role of the unconscious in film from Buñuel to Kieślowski (funded from the Irish Research Council for the Humanities and Social Sciences). 